\title{
Esophageal Fistula following Anterior Cervical Discectomy and Fusion in Traumatic Cervical Injury Cases: A Review
}

\author{
Rajesh K. Meena ${ }^{1} \quad$ Ramesh S. Doddamani ${ }^{1} \quad$ Dattaraj P. Sawarkar ${ }^{1} \quad$ Pankaj K. Singh $^{1} \quad$ Deepak Agarwal $^{1}$ \\ ${ }^{1}$ Department of Neurosurgery, All India Institute of Medical \\ Address for correspondence Ramesh S. Doddamani, MCh, \\ Sciences, New Delhi, India \\ Department of Neurosurgery, All India Institute of Medical Sciences \\ (AlIMS), New Delhi 110049, India (e-mail: drsdramesh@gmail.com).
}

Indian J Neurotrauma:2020;17:33-36

\begin{abstract}
Background Esophageal fistula is a very rare complication associated with anterior approaches to the cervical spine. Timely diagnosis and early intervention are associated with favorable outcomes. There is a dilemma in the literature for its optimal management. In this review article, we will discuss the management dilemmas in patients with esophageal perforation along with an illustrative case.

Material and Methods A 24-year-old male patient operated for C5-6 fracture dislocation, presented with esophageal fistula 1 month after surgery. Investigations were done to localize the site of fistula but definitive site of leak could not be identified.

Keywords

- trauma

- anterior

- cervical spine

- esophageal

- fistula Patient was counselled regarding possible surgical intervention; however, patient was opted for conservative treatment.

Conclusion Esophageal fistula is a very rare occurrence. Because of varied clinical presentation and frequent delay in diagnosis, a very high index of suspicion should always be kept in mind while evaluating a patient postoperatively for dysphagia. Timely diagnosis and early intervention are key to a successful outcome.
\end{abstract}

\section{Introduction}

Anterior approaches to the cervical spine are frequently used to treat cervical spine injuries and cervical spondylosis. Although surgical outcomes are satisfactory in vast majority of the patients, complications may occur on rare occasions. Most of these complications are transient and self-limited, but in some unfortunate instances, they may lead to significant morbidity and mortality. ${ }^{1-5}$ Esophageal perforation is one such devastating iatrogenic complication. Reported incidence in the literature varies from 0.04 to $0.25 \%{ }^{2,6}$ Mortality from esophageal perforation varies from 9 to $45 \% .^{1,2,6}$ This complication has been reported to occur either intraoperatively, perioperatively, or in delayed fashion, that is, weeks, months, or years after surgery. Clinical presentation of this complication is highly variable. Patient may be asymptomatic or may present with dysphagia, odynophagia, local soft tissue infection, deep infection with hardware failure, osteomyelitis, pseudo arthrosis, and can be fatal at times with sepsis/infectious mediastinitis. . $^{1-4,7-9}$ As morbidity and mortality from this complication is high early diagnosis and prompt institution of therapy is essential for successful outcome. In this review article, we will discuss the management dilemmas in patients with esophageal perforation along with an illustrative case.

\section{Case Illustration}

A 24-year-old male patient was admitted to our hospital with neck pain and weakness in all four limbs following a road traffic accident. Imaging showed of C5-6 fracture dislocation (-Fig. 1A and B ). On neurological examination motor power in bilateral upper limb was three-fifths with hand grip of $50 \%$. Lower limb power was $0 / 5$ with complete absence of sensation below C7 level. He underwent 

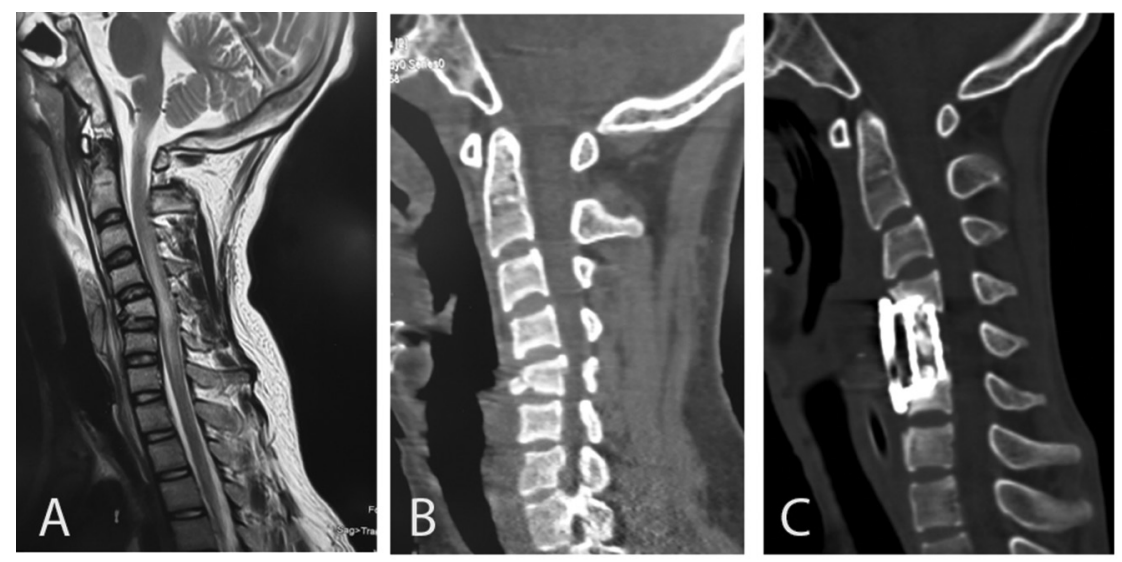

Fig. 1 Preoperative scans of the patient. (A) Magnetic resonance imaging (MRI) of cervical spine-sagittal view; (B) computed tomographic (CT) scan of cervical spine-sagittal view. Postoperative CT scan of the patient; (C) sagittal view showing C5 corpectomy and mesh cage placement with anterior cervical plating.
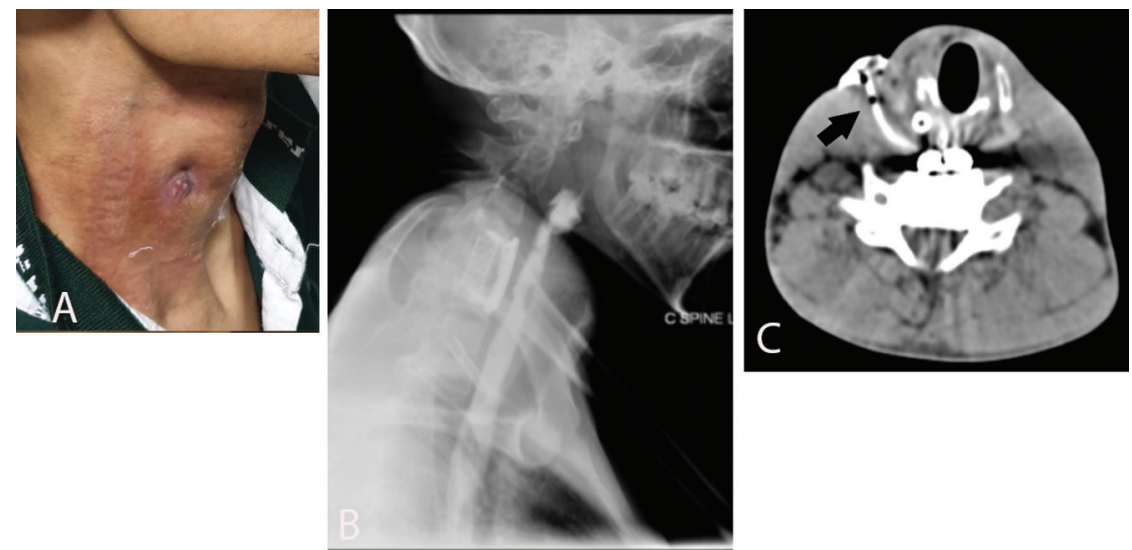

Fig. 2 Development of Esophageal fistula 1 month after surgery. (A) Clinical photograph of the patient; (B) barium swallow study showing no definite evidence of fistula; (C) modified contrast swallow with sinogram showing sinus tract (arrow) up to the cervical esophagus.

C5 corpectomy and mesh cage placement with anterior cervical plating (-Fig. 1C). Postoperative period was uneventful, with neurological status same as in preoperative period and he was discharged home on postoperative day 6. On follow-up period, 1 month after surgery, he started complaining of progressively increasing dysphagia. He also complained of swelling and redness in the neck with discharging sinus at the operative site, findings suggestive of esophageal fistula ( - Fig. 2A). There was no history of fever and hematological investigations were within normal limits. Contrast enhanced computed tomography (CT) scan of the neck and ultrasound of the neck were done that showed $4 \mathrm{~cm} \times 2.5 \mathrm{~cm}$ collection in the neck, lateral to the right thyroid lobe with few air foci showed the same findings. Barium swallow test was done to identify the site of leak, but was negative (-Fig. 2B). Repeat contrast swallow with sinogram was done which showed a collection in the right side of the neck that was communicating with the cervical esophagus (esophageal cutaneous fistula; - Fig. 2C). Esophagoscopy/endoscopy was negative. Patient was counselled regarding the surgery for the management of esophageal fistula; however, patient refused any further surgical intervention and lost to follow-up.

\section{Discussion}

Esophageal fistula following anterior approaches to the cervical spine is of rare occurrence. Various predisposing factors have been reported in the literature, without any conclusive evidence for its occurrence, as follows:

- Esophageal or spine trauma: compression of vertebral fracture segments, as well as traumatic stretching and twisting, could cause esophageal wall injuries may be due to ischemia.

- History of esophageal diverticula/reflux disease.

- Lack of serosa in esophagus, thinner posterior wall and poorer blood supply for muscle layers: at Lannier's triangle (formed by the cricopharyngeus and constrictor pharyngeus muscle at the $\mathrm{C} 5$ and $\mathrm{C} 6$ levels), the posterior pharyngeal wall is very thin, covered only by fascia. ${ }^{6}$

- Use of Sharp retractors and instruments: retractors should be placed below the longus colli muscles medially.

- Use of high speed drill as well as during the instrumentation phase.

- Pressure sores caused by the metallic implant and its microtrauma effect on the esophagus.

- Suboptimal placement of screws.

- Chronic, erosive process caused by anterior instrumentation. 
- Failed hardware as well as hardware that was prominent and causing mass effect and eventual erosion.

- Thermal injury: if electrotomes were used improperly electric currents can injure esophagus by the conduction of metal hooks, retractors, or other conductors.

Esophageal perforation may manifest itself, intraoperatively with recognized injury or anytime from the early postoperative period to years later, with varied clinical presentation. ${ }^{1-4,7-9}$ Most commonly patients presented with dysphagia and odynophagia in a literature review by Halani et al. ${ }^{2}$ Postoperatively, any patient presenting with neck swelling, persistent neck or throat pain, crepitus in the neck, wound leak, cervical abscess, fever, septicemia, mediastinitis, or unexplained fevers should alert the clinician to the possibility of esophageal perforation. ${ }^{10,11}$ As dysphagia is the most common presenting symptom, any patient with difficulty in swallowing after an anterior surgical intervention to the cervical spine, even years later, should undergo a thorough workup for a possible esophageal perforation.

A gamut of investigations were used to diagnose the site of esophageal perforation; however, only $72 \%$ of the patients had positive findings on an imaging study. ${ }^{11-15}$

- X-ray neck can be useful for detecting air around the screw, plate site, or the graft.

- Contrast swallowing studies (Gastrografin, methylene blue, and barium) can delineate the site of leak in majority of the patients. Additionally, endoscopy/esophagoscopy can demonstrate the site of leak.

- High resolution magnetic resonance imaging (MRI) can be useful for diagnosis of cervical osteomyelitis with or without epidural abscess.

- Flexion and extension radiographs along with CT scan can be used to look for bony fusion and need for posterior fixation.

In our case, esophageal perforation may have resulted either from intraoperative instrument manipulation or due to sub optimal placement of $\mathrm{C} 4$ screw and plate causing microtrauma and erosions in the cervical esophagus. Multiple diagnostic modalities are available to look for the possible site of leak; however, the results are often negative as also seen in our case.

\section{Treatment}

Several treatment options are available for managing esophageal injury; however, optimal treatment remains elusive, owing to its rarity. There is a dilemma in the literature, whether these injuries needs to be treated aggressively or a conservative approach is more optimal. ${ }^{1-6,16-18}$ As morbidity and mortality associated with esophageal perforation is high, timely diagnosis and early institution of appropriate treatment is of paramount importance for successful outcome.

- If the injury is recognized intraoperatively, primary repair with or without tissue reinforcement (omental, pectoral or sternocleidomastoid [SCM] flaps), short-term esophageal rest (via nasogastric tube placement), and injectable antibiotics is preferred.
- If the injury is recognized in the early postoperative period (small rent, contained well within the cervical spine and with minimal symptoms), nasogastric tube placement and injectable antibiotics may suffice.

- If the injury is recognized in the early postoperative period (large rent, with greater symptoms, and more likelihood of developing mediastinitis and sepsis) or with delayed presentation, reexploration of wound is advocated with following options:

- Reexploration with primary closure often difficult owing to inflamed esophageal wall.

- Reexploration with reinforced tissue closure with flaps from SCM, pectoralis, infrahyoid, omohyoid, latissimus dorsi, longus colli, radial forearm muscles, or the omentum. SCM is the most commonly used muscle flap as it is anatomically close to the esophagus, easy to harvest, pliable, and has great vascular supply. After exposing the entire medial and lateral surface of the SCM, with preservation of spinal accessory nerves an inferiorly pedicled flap is interposed between the esophagus and the spine..$^{19}$ This is followed by suturing the distal end of the flap to the prevertebral tissue on the opposite side to hold the flap in place during the healing process.

- Wound drainage (controlled esophagocutaneous fistula via T-tube placement).

- Exclusion and diversion (end-cervical esophagostomy and gastrostomy).

Role of removal of anterior hardware: Halani et al in a systematic review of the literature on esophageal perforation after anterior cervical surgery found that the average number of esophageal repair attempts per patient was $1.54(n=96){ }^{2}$ Sixty-three patients achieved complete closure of esophageal perforation after first repair attempt, while 29 patients required two or more additional surgeries before definitive closure was achieved. In 21 out of 29 patients who required more than two surgeries, removal of anterior hardware was done for the complete resolution of the perforation. Therefore, removal of the anterior hardware plays a pivotal role in healing of recurrent esophageal perforations.

Another major concern in the patients with esophageal perforation is nutrition, as the patients were kept nil per mouth till healing of perforation occurs. Healing usually takes 3 to 12 weeks. Nutrition was provided with the assistance of nasogastric tube and/or gastrostomy or jejunostomy. Many studies have demonstrated that feeding by nasal tube alone for prolonged periods is insufficient to meet the nutritional requirement, therefore advocating the placement of jejunostomy or gastrostomy. ${ }^{10,12}$ To prevent negative nitrogen balance, compound amino acids can be administered intravenously which is favorable to wound healing. Halani et al reported that the average time to oral intake following repair of esophageal perforation was 30.2 days (range: 4-188 days) and it was significantly longer for the patient who managed conservatively (average, 68 days). ${ }^{2}$ Therefore, managing nutrition in patients who were managed conservatively is more challenging. 
Traditionally, conservative measures, such as prolonged fasting, broad spectrum antibiotics, and nutritional support have been used to treat esophageal fistula. However, conservative treatment itself has failure rates and it is very difficult to determine accurately that patients would fail conservative treatment on initial diagnostic tests. In addition to challenges in maintaining the nutritional status, possibility of abscess formation and osteomyelitis, mortality of 18 to $20 \%$ have been reported for patients on conservative treatment. Therefore more and more studies are favoring early surgical intervention for the management of esophageal fistulas. ${ }^{1-6}$

\section{Conclusion}

Esophageal perforation following anterior approaches to the cervical spine is a rare, but potentially life-threatening complication, if not managed appropriately. C5-6 level seems to be the most susceptible level to esophageal injury. Utmost care should be taken during exposure and spinal instrumentation to avoid contact with the esophagus. Awareness, early recognition of injury, and appropriate management individualized on a case to case basis is of paramount importance to improve outcome and quality of life. Lastly, any patient with dysphagia after an anterior approach to the cervical spine, even years later, should receive thorough workup for a possible esophageal perforation.

\section{Conflict of Interest}

None.

\section{Funding Information}

None.

\section{Financial Support}

None.

\section{References}

1 Dakwar E, Uribe JS, Padhya TA, Vale FL. Management of delayed esophageal perforations after anterior cervical spinal surgery. J Neurosurg Spine 2009;11(3):320-325

2 Halani SH, Baum GR, Riley JP, et al. Esophageal perforation after anterior cervical spine surgery: a systematic review of the literature. J Neurosurg Spine 2016;25(3):285-291

3 LeeTS, Appelbaum EN, Sheen D, Han R, Wie B. Esophageal perforation due to anterior cervical spine hardware placement: case series. Int J Otolaryngol 2019;2019:7682654

4 Krishnan P, Kartikueyan R, Patel SM, Bose PP, Mukherjee KK. Two cases of esophageal injury following anterior cervical discectomy and fusion: one overt and one covert. Ann Neurosci 2017;24(2):126-130
5 Yang S-Y, Lee S-B, Cho K-S. Delayed esophagus perforation after anteriorcervical spine surgery. Korean J Neurotrauma 2015;11(2):191

6 Tasiou A, Giannis T, Brotis AG, et al. Anterior cervical spine surgery-associated complications in a retrospective case-control study. J Spine Surg 2017;3(3):444-459

7 Paradells VR, Pérez JB, Vicente FJ, Florez LB, de la Viuda MC, Villagrasa FJ. Esophageal, pharyngeal and hemorrhagic complications occurring in anterior cervical surgery: Three illustrative cases. Surg Neurol Int 2014;5(4, Suppl 3) :S126-S130

8 Patel NP, Wolcott WP, Johnson JP, et al. Esophageal injury associated with anterior cervical spine surgery. Surg Neurol 2008;69(1):20-24, 24

9 Perrone $\mathrm{O}$, Tassi V, Mattioli B, et al. Pharyngo-oesophageal perforation following anterior cervical discectomy and fusion: management and results. Eur J Cardiothorac Surg 2017;51(1):160-168

10 Darbari A, Suryavanshi A, Tandon S, Chandra G, Singh PK. Non malignant tracheo-esophageal fistula: Our experience. Indian J Thorac Cardiovasc Surg 2005;21(4):272-276

11 Newhouse KE, Lindsey RW, Clark CR, Lieponis J, Murphy MJ. Esophageal perforation following anterior cervical spine surgery. Spine 1989;14(10):1051-1053

12 Dabija MG, Iliescu BF, Andronic D, Popescu C, Ianovici N. Rare complication of the cervical spine trauma-traumatic esophageal fistula: case report and review of the literature. Rev Med Chir Soc Med Nat Iasi 2014;118(3):683-687

13 Phommachanh V, Patil YJ, McCaffrey TV, Vale F, Freeman TB, Padhya TA. Otolaryngologic management of delayed pharyngoesophageal perforation following anterior cervical spine surgery. Laryngoscope 2010;120(5):930-936

14 Sharma RR, Sethu AU, Lad SD, Turel KE, Pawar SJ. Pharyngeal perforation and spontaneous extrusion of the cervical graft with its fixation device: a late complication of C2-C3 fusion via anterior approach. J Clin Neurosci 2001;8(5):464-468

15 Solerio D, Ruffini E, Gargiulo G, et al. Successful surgical management of a delayed pharyngo-esophageal perforation after anterior cervical spine plating. Eur Spine J 2008;17(Suppl 2) : S280-S284

16 Volkow-Fernández P, Islas-Muñoz B, Santillán-Doherty $\mathrm{P}$, Estrada-Lobato E, Alva-López L, Ávila-Ramírez J. Successive complications after anterior cervical fixation: pharyngoesophageal diverticulum, fistulization, and cervical spondylitis by Streptococcus milleri - case report and literature review. J Med Case Reports 2019;13(1):129

17 Wang J, Shi L, Chen H, Yuan W. Esophageal Perforation in a Cervical Fracture Patient With Progressed Ankylosing Spondylitis: Case Report and Review of the Literature. Spine 2016;41(22):E1364-E1367 Review

18 Yuan H, Ding H, Hu L, Buser Z, Zhao H, Li X. Treatment for early postoperative esophageal fistula complicated with anterior cervical surgery. J Orthop Surg (Hong Kong) 2017;25(1):2309499016684418

19 Al-Mourgi M. Sternocleidomastoid flap for repair of pharyngocutaneous fistula following anterior cervical spine surgery. Saudi J Health Sci 2015;4(3):199 